\title{
Triplet Dynamic Nuclear Polarization of Guest Molecules through Induced Fit in a Flexible Metal-Organic Framework
}

\author{
Saiya Fujiwara, ${ }^{[a]}$ Naoto Matsumoto, ${ }^{[a]}$ Koki Nishimura, ${ }^{[a]}$ Nobuo Kimizuka, ${ }^{[a]}$ Kenichiro Tateishi, ${ }^{[b]}$ \\ Tomohiro Uesaka, ${ }^{[b]}$ and Nobuhiro Yanai ${ }^{\star[a, c]}$
}

\begin{abstract}
Dynamic nuclear polarization utilizing photoexcited triplet electrons (triplet-DNP) has great potential for room-temperature hyperpolarization of nuclear spins. However, the polarization transfer to molecules of interest remains a challenge due to the fast spin relaxation and weak interaction with target molecules at room temperature in conventional host materials. Here, we demonstrate the first example of DNP of guest molecules in a porous material at around room temperature by utilizing the induced-fit-type structural transformation of a crystalline yet flexible metal-organic framework (MOF). In contrast to the usual hosts, ${ }^{1} \mathrm{H}$ spin-lattice relaxation time becomes longer by accommodating a pharmaceutical model target 5fluorouracil as the flexible MOF changes its structure upon guest accommodation to maximize the host-guest interactions. Combined with triplet-DNP and cross-polarization, this system realizes an enhanced ${ }^{19} \mathrm{~F}-\mathrm{NMR}$ signal of guest target molecules at around room temperature.
\end{abstract}

Dynamic nuclear polarization (DNP) is the method for polarization transfer from electron spins to nuclear spins to overcome the limited sensitivity of nuclear magnetic resonance (NMR) and magnetic resonance imaging (MRI). ${ }^{[1-11]}$ In particular, DNP utilizing photoexcited triplet electrons (triplet-DNP) has great potential as one of the methods for nuclear hyperpolarization at room temperature. ${ }^{[12-15]}$ In general, triplet-DNP has been conducted in host matrices doped with polarizing agent molecules, where the photoexcited polarizing agent undergoes spin-selective intersystem crossing (ISC) and provides a non-equilibrium electron spin polarization in the triplet sublevels independent of temperature (Figure 1a). Then, the hyperpolarization of triplet electrons is transferred to nuclei by microwave irradiation under magnetic field sweep (integrated solid effect, ISE). ${ }^{[12]}$

In recent years, many efforts have been devoted to developing new molecules, materials and methodologies to advance triplet-DNP into biological and medical applications. ${ }^{[16-21]}$ However, while a few studies have achieved hyperpolarization of biologically-relevant molecules using triplet-DNP, those systems often require low temperatures $(\sim 100 \mathrm{~K}) \cdot{ }^{[16,19,22]}$ The reason for this, despite the temperature-independent polarization source being a key feature for triplet-DNP, is mainly that the polarization source

[a] S. Fujiwara, N. Matsumoto, K. Nishimura, Prof. N. Kimizuka, Prof. N. Yanai

Department of Applied Chemistry, Graduate School of Engineering,

Center for Molecular Systems (CMS), Kyushu University 744 Moto-

oka, Nishi-ku, Fukuoka 819-0395, Japan

E-mail: yanai@mail.cstm.kyushu-u.ac.jp

[b] Dr. K. Tateishi, Dr. T. Uesaka

Cluster for Pioneering Research, RIKEN,

RIKEN Nishina Center for Accelerator-Based Science, Wako, Saitama 351-0198, Japan

[c] Prof. N. Yanai

PRESTO, JST, Honcho 4-1-8, Kawaguchi, Saitama 332-0012, Japan and target molecules need to be dispersed in the low-temperature glasses to stop the molecular motions for the effective polarization transfer. Moreover, since it is difficult for the natively fluid hosts to fix the dynamics of guest molecules, the resulting mixtures often show dropping the nuclear spin-lattice relaxation time $\left(T_{1}\right)$ even at such low temperatures. ${ }^{[16,22]}$ The use of target molecules itself as the host matrix can be a possible solution, ${ }^{[19,23-24]}$ but the applicable target molecules are severely limited due to the strict requirements such as the long $T_{1}$ at room temperature and the good dispersibility of polarizing agents.

Another promising approach is to confine the polarization source and target molecules in the nanopores of porous materials, which is expected to enable hyperpolarization of a variety of guest molecules. Unique DNP systems based on various nanoporous materials have been developed, ${ }^{[8,25-28]}$ and our group has also reported triplet-DNP of metal-organic frameworks (MOFs). ${ }^{[29]}$ However, there have been no examples of polarization transfer to target guest molecules in nanoporous materials at around room temperature. This is because the mobility of the guest molecules could not be sufficiently suppressed near room temperature, and the polarization transfer did not occur efficiently. ${ }^{[30-31]}$

Here, we report the first example of DNP of guest molecules in a porous material near room temperature through induced fit in a crystalline yet flexible MOF (Figure 1b). MOFs have been extensively studied as promising porous materials, ${ }^{[2-37]}$ and the guest-responsive structural change, also called as the 'breathing' behavior, is one of the most fascinating features of MOFs not observed in conventional porous materials. ${ }^{[38-39]} \mathrm{A}$ representative breathing MOF $\left[\mathrm{Al}\left(\mu_{2}-\mathrm{OH}\right)(\mathrm{BDC})\right]_{\mathrm{n}}(\mathrm{MIL}-53 ; \mathrm{BDC}=$ terephthalate) has been demonstrated to form the prominent host-guest

a)

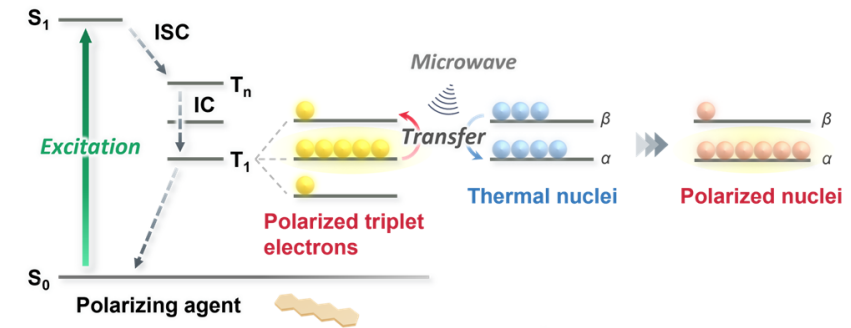

b)

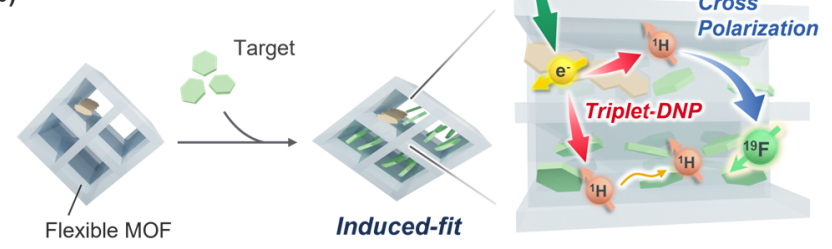

Figure 1. a) Typical scheme of triplet-DNP. ISC: intersystem crossing, IC: internal conversion. b) Schematic representation of the hyperpolarization of target nuclei in a flexible MOF at around room temperature by triplet-DNP and cross-polarization. 
interactions with various guest molecules by adjusting its onedimensional channel structure. ${ }^{[40-42]}$ We introduced a polarizing agent 5,12-diazatetracene $(\mathrm{DAT})^{[43]}$ and a benchmark pharmaceutical model compound 5-fluorouracil (5-FU) $)^{[16,44]}$ into partially deuterated MIL-53 (denoted as D-MIL-53, Figure 2a). DMIL-53 showed an induced-fit-type structural change upon 5-FU uptake, and notably, this system showed an elongation of $T_{1}$ by the guest accommodation, which is the opposite trend from the conventional glass systems. The triplet-DNP and subsequent cross-polarization (CP) sequences successfully enhanced the ${ }^{19} \mathrm{~F}-\mathrm{NMR}$ signal of guest 5-FU at around room temperature. a)

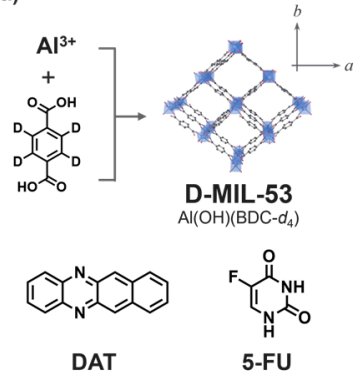

c)

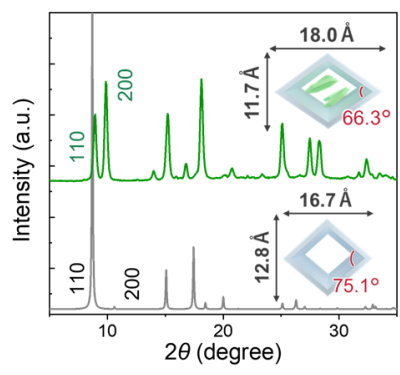

b)

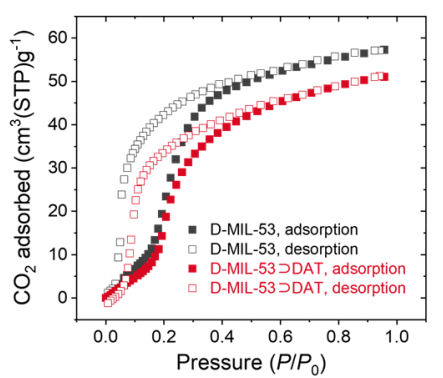

d)

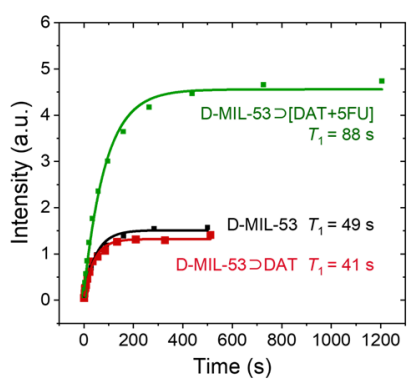

Figure 2. a) Chemical structures of host D-MIL-53, polarizing agent DAT, and target 5-FU. b) $\mathrm{CO}_{2}$ adsorption isotherms at room temperature for D-MIL-53 and D-MIL-53دDAT. (c) PXRD patterns of D-MIL-53د[DAT+5-FU] (green) and simulated MIL-53 in the LP phase (gray). ${ }^{[40]}$ Schematic representations of the host-guest structures are also shown. The lozenge diagonal lengths correpond to the cell parameters $a$ and $b$ as summarized in Table S1. (d) Comparison of saturation-recovery curves of D-MIL-53 with/without the guest molecules at 400 $\mathrm{MHz}$, room temperature. The recovery curves are fitted with single-exponential functions.

The breathing MOF MIL-53 was synthesized by the oxalic acid modulation method (Scheme S1). ${ }^{[45]}$ To suppress the spinlattice relaxation of the ${ }^{1} \mathrm{H}$ nuclei in MIL-53 due to the rotation of phenyl rings, we constructed phenyl-deuterated MIL-53 (denoted as D-MIL-53) using the deuterated ligand BDC- $d_{4}$. The air-stable and soluble polarizing agent DAT was synthesized and introduced into the nanochannels (pore size $=8.5 \times 8.5 \AA$, large pore (LP) phase) of D-MIL-53. In our previous report using prototypical MOF ZIF-8, ${ }^{29]}$ polarizing agent was kinetically trapped by accelerating the MOF crystallization, whereas D-MIL53 could readily adsorb DAT post-synthetically to form a MOFpolarizing agent composite (denoted as D-MIL-53دDAT).

The aggregation of polarizing agents has been known to significantly degrade the polarization ability in triplet-DNP. ${ }^{[15]}$ The dispersion of DAT inside D-MIL-53 was confirmed by UV-Vis absorption measurements (Figure S7). D-MIL-53دDAT showed a vibronic $\pi-\pi^{*}$ absorption band with peaks at $515.5,480.5$, and $450.0 \mathrm{~nm}$. These peaks were clearly sharpened and blue-shifted compared to those of bulk DAT solids. This trend was similar to that of DAT doped in $p$-terphenyl crystals in which a significant enhancement of ${ }^{1} \mathrm{H}$ spin polarization was reported, ${ }^{[43]}$ indicating that DAT molecules are well-dispersed in D-MIL-53. The amount of DAT in D-MIL-53 was optimized to prevent the aggregation of DAT molecules. From UV-Vis absorbance after digesting D-MIL53DDAT (Figure S8), the number of DAT molecules per unit cell of D-MIL-53 was estimated to be 0.006 .

The composite D-MIL-53دDAT showed a similar powder Xray diffraction (PXRD) pattern to that of hydrated D-MIL-53 (narrow pore (NP) phase) that adsorbs water molecules in air (Figure S9). Thermogravimetric analysis (TGA) curves also showed similar weight losses at $25-100^{\circ} \mathrm{C}$ for D-MIL-53 and DMIL-53دDAT due to the dehydration process (Figure S10). ${ }^{[40]}$ The $\mathrm{CO}_{2}$ adsorption isotherms of dehydrated D-MIL-53 and D-MIL53DDAT showed a multi-step adsorption (LP to NP phase) and hysteresis was observed in the desorption process (NP to LP phase, Figure $2 b$ ). These behaviors are characteristic of the unconstrained MIL-53 and are consistent with previous studies, ${ }^{[46-}$ ${ }^{47]}$ confirming that the breathing behavior of MIL-53 is well retained after the DAT accommodation.

The target 5-FU molecules were sublimated and introduced into the nanochannels of D-MIL-53دDAT. Note that the excess bulk 5-FU was removed until the PXRD peaks of bulk 5-FU disappeared (Figure S11). PXRD patterns showed a significant change of the host crystal structure, resulting in the formation of the host-guest composite described as D-MIL-53つ[DAT+5-FU]. UV-Vis absorption spectrum of this composite showed slightly broad and blue-shifted $\pi-\pi^{*}$ absorption peaks (512.5, 479.0, $447.5 \mathrm{~nm}$, Figure S12). This small change would be attributed to the environmental changes surrounding DAT due to the presence of 5-FU. It rather indicates DAT molecules were not decomposed during the introduction of 5-FU thanks to their high stability. The TGA curve of D-MIL-53د[DAT+5-FU] showed $39 \%$ weight loss at $190-310^{\circ} \mathrm{C}$ for the adsorbed 5-FU (Figure S13). This weight loss corresponds to 1.065 -FU molecules per unit cell of the host, which is in good agreement with the number obtained by the elemental analysis (1.08).

The structural transformation of the host was further characterized by Le Bail fitting of the PXRD pattern (Figure S14). The fitting result suggests that the crystal system of D-MIL$53 \supset[D A T+5-F U]$ remained the same as the original orthorhombic, but the lozenge-shaped 1-D channels were distorted and the angle of the lozenge was decreased $\left(66.3^{\circ}\right)$ compared to the guest-free MIL-53 $\left(75.1^{\circ}\right) .^{[40]}$ This indicates the induced-fit shrinkage of host D-MIL-53 to guest 5-FU (Figure 2c). The obtained lozenge diagonal lengths of D-MIL-53د[DAT+5-FU] were similar to those reported for MIL-53 loaded with one equivalent of quinone molecules in the same orthorhombic system (Table S1). ${ }^{[42]}$ Considering the similarity of the molecular structure and the comparable number per unit cell of the host, it is suggested that 5-FU molecules are trapped in D-MIL-53 in a similar fashion for MIL-53 5 quinone ${ }^{[48]}$ through $\pi-\pi$ interactions with both neighboring 5-FU molecules and benzene rings of BDC$d_{4}$ linkers.

The nuclear spin relaxation property was evaluated by measuring spin-lattice relaxation time $T_{1}$ of ${ }^{1} \mathrm{H}$ nuclei (Figure $2 \mathrm{~d}$ ). The original ${ }^{1} \mathrm{H} T_{1}$ value of D-MIL-53 was $49 \mathrm{~s}$, but after the addition of $0.6 \mathrm{~mol} \% \mathrm{DAT}$, the ${ }^{1} \mathrm{H} T_{1}$ value slightly decreased to $41 \mathrm{~s}$. Such reduction in ${ }^{1} \mathrm{H} T_{1}$ by the guest accommodation has also been observed in the previous triplet-DNP systems. ${ }^{[16,22,29]}$ 
On the other hand, interestingly, D-MIL-53د[DAT+5-FU], where the host MOF underwent the induced-fit to the guest 5-FU, showed a large increase in ${ }^{1} \mathrm{H} T_{1}$ value to $88 \mathrm{~s}$. These results suggest that the unfavorable molecular motions are effectively suppressed in the host-guest composite even at room temperature, which should be beneficial for polarization transfer.

The intact electron spin polarization properties of DAT in the D-MIL-53つ[DAT+5-FU] composite were confirmed by timeresolved electron spin resonance (ESR) measurements at room temperature (Figure S1, S2). The ESR spectrum of D-MIL$53 \supset[D A T+5-F U]$ showed the typical line shape for the polarized triplet excited state of DAT (Figure 3a). The population rates in the three sublevels of the triplet state were estimated by using the EasySpin toolbox in MATLAB. ${ }^{[49]}$ As expected from the comparison of UV-Vis absorption spectra, the zero-field splitting parameters $(|D|=1616 \mathrm{MHz},|E|=164 \mathrm{MHz})$ and relative zerofield populations $\left(P_{\mathrm{x}}: P_{\mathrm{y}}: P_{\mathrm{z}}=0.706: 0.110: 0.184\right)$ of D-MIL$53 \supset[\mathrm{DAT}+5-\mathrm{FU}]$ were similar to those of DAT doped in $p$ terphenyl (Table S2). ${ }^{[43]}$ The triplet polarization lifetime was $6.5 \mu \mathrm{s}$ (Figure $3 b$ ), which is long enough for the polarization transfer through the ISE sequence.

a)

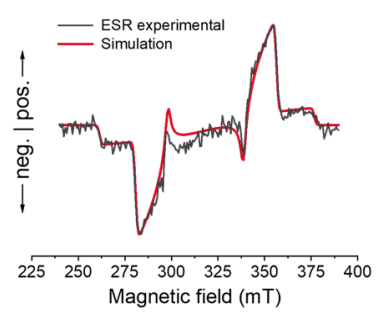

c)
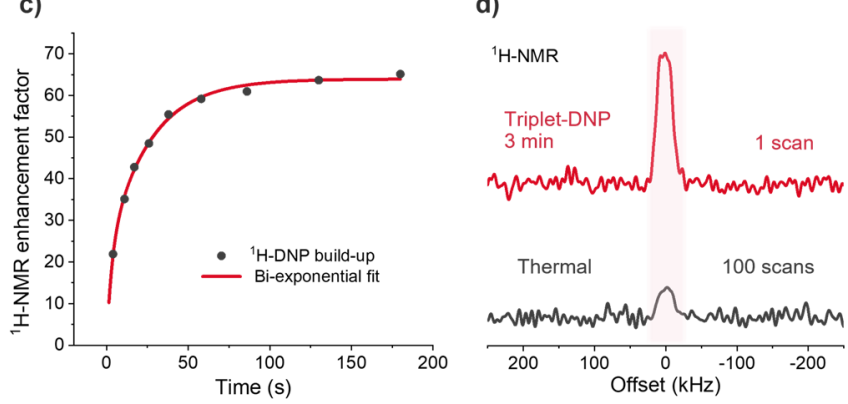

Figure 3. a) Time-resolved ESR spectrum of D-MIL-53つ[DAT+5-FU] just after photoexcitation at $527 \mathrm{~nm}$ and simulated spectrum. Fitting parameters are summarized in Table S2. b) Decay of the ESR peak at $353 \mathrm{mT}$ under pulsed excitation at $527 \mathrm{~nm}$ and the result of single-exponential fitting. c) ${ }^{1} \mathrm{H}$-DNP buildup curve of D-MIL-53 $[\mathrm{DAT}+5-\mathrm{FU}]$ at around room temperature and the result of double-exponential fitting. Fitting parameters are shown in Table S3. The enhancement factor was calculated by comparing the peak area with thermal equilibrium. d) $28.2 \mathrm{MHz}{ }^{1} \mathrm{H}-\mathrm{NMR}$ signals of under thermal conditions (100 scans) and after triplet-DNP (ISE sequence for $3 \mathrm{~min}, 1 \mathrm{scan}$ ) at around room temperature.

Triplet-DNP in the D-MIL-53つ[DAT+5-FU] was conducted by using a home-built spectrometer with an ISE sequence at 0.66 $\mathrm{T}$ and room temperature (Figure S3, S4). The polarizing agent DAT was photoexcited by a pulsed $527 \mathrm{~nm}$ laser, followed by 17.6 $\mathrm{GHz}$ microwave irradiation for $30 \mu$ s with field sweep for polarization transfer to ${ }^{1} \mathrm{H}$ nuclei. After repeating this process, the polarized sample was shuttled into the NMR coil above the resonator within $1 \mathrm{~s}$ to obtain the ${ }^{1} \mathrm{H}-\mathrm{NMR}$ signal. Since the accumulation of ${ }^{1} \mathrm{H}$ spin polarization competes with spin-lattice relaxation, the enhancement factor of the ${ }^{1} \mathrm{H}-\mathrm{NMR}$ signal saturates as the repetition of the ISE sequence (Figure 3c). After the triplet-DNP process for $3 \mathrm{~min}$, the D-MIL-53د[DAT+5-FU] showed the 64-fold enhancement of the ${ }^{1} \mathrm{H}-\mathrm{NMR}$ signal at around room temperature (Figure $3 d$ ).

The enhanced ${ }^{1} \mathrm{H}$ spin polarization was successfully transferred to ${ }^{19} \mathrm{~F}$ nuclei of target guest 5 -FU by using the typical $\mathrm{CP}$ sequence. The strengths of the magnetic fields irradiated to ${ }^{1} \mathrm{H}$ and ${ }^{19} \mathrm{~F}$ spins were kept at about $50 \mathrm{kHz}$, and the contact time was set to $0.5 \mathrm{~ms}$. After the $\mathrm{CP}$ sequence following the tripletDNP for $100 \mathrm{~s}$ (Figure 4a), the D-MIL-53د[DAT+5-FU] composite clearly showed the 38-fold enhanced ${ }^{19} \mathrm{~F}-\mathrm{NMR}$ signal (Figure $4 \mathrm{~b}$ ). This result provides the first example of DNP of guest molecules in a porous host at around room temperature. Theoretically, the maximum ${ }^{19} \mathrm{~F}-\mathrm{NMR}$ enhancement factor obtained by $\mathrm{CP}$ is defined as the product of the ${ }^{1} \mathrm{H}-\mathrm{NMR}$ enhancement factor and the ratio of the gyromagnetic ratio $\left(=\gamma_{H} / \gamma_{F}\right) \cdot{ }^{[30]}$ Therefore, the optimal CP in D-MIL-53כ[DAT+5-FU] can result in 67 -fold ${ }^{19} \mathrm{~F}$ NMR enhancement at maximum, and thus the experimental efficiency of the CP was estimated to be about $57 \%$. This value is comparable to that of the reported $o$-terphenyl glass system at low temperature (ca. $70 \%, 120 \mathrm{~K}$ ), ${ }^{[16]}$ and the optimization of Hartmann-Hahn matching and pulse sequence design (e.g. variable-amplitude $\mathrm{CP}$ ) would lead to higher enhancements.

a)

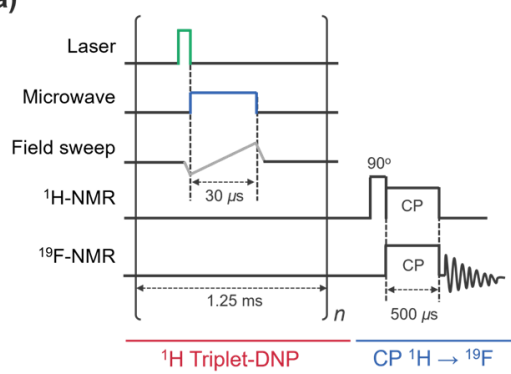

b)

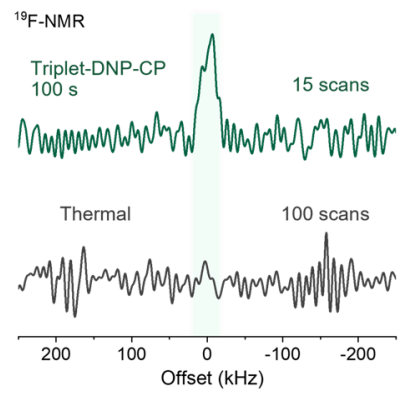

Figure 4. a) Sequence of the triplet-DNP-CP process. b) $26.5 \mathrm{MHz}{ }^{19} \mathrm{~F}-\mathrm{NMR}$ signals of D-MIL-53د[DAT+5-FU] under thermal conditions (100 scans) and after triplet-DNP-CP (ISE sequence for $100 \mathrm{~s}$ followed by CP, 15 scans) at around room temperature.

In conclusion, the hyperpolarization of guest molecules in a porous host material was achieved for the first time at around room temperature. The crystalline yet flexible MOF incorporated the guest target molecules with changing its pore structure to adjust the host-guest interactions. The unique phenomenon was observed in which the ${ }^{1} \mathrm{H}$ spin-lattice relaxation time $T_{1}$ was rather extended by the guest incorporation. In addition, the strong hostguest interaction suppresses the mobility of the guest target molecules sufficiently to realize the polarization transfer to guest 
molecules through triplet-DNP and CP even at around room temperature. Although the current work focused only on the benchmark model target 5-FU, there are diverse approaches using MOFs such as the chemical functionalization and the tunable guest/stimuli-responsive behavior, ${ }^{[50]}$ which would expand the versatility of the current concept for other molecules of interest. ${ }^{[51]}$ We believe that this strategy exploiting the chemistry of soft porous crystals will provide new perspectives for the development of innovative materials and technologies in room-temperature DNP.

\section{Acknowledgements}

This work was partly supported by the JST-PRESTO program on "Creation of Life Science Basis by Using Quantum Technology" (grant number: JPMJPR18GB), JSPS KAKENHI (grant numbers: JP19J21421, JP2OH02713, JP2OK21211, JP2OH05676, and JP21J13049), The Shinnihon Foundation of Advanced Medical Treatment Research, the Innovation inspired by Nature Program of Sekisui Chemical Co. Ltd., RIKEN-Kyushu Univ Science and Technology Hub Collaborative Research Program, the RIKEN Cluster for Science, Technology and Innovation Hub (RCSTI), and the RIKEN Pioneering Project "Dynamic Structural Biology".

Keywords: dynamic nuclear polarization $\cdot$ nuclear magnetic resonance $\cdot$ photoexcited triplet $\cdot$ metal-organic frameworks • soft porous crystals

[1] A. W. Overhauser, Phys. Rev. 1953, 92, 411-415.

[2] T. R. Carver, C. P. Slichter, Phys. Rev. 1953, 92, 212-213.

[3] D. A. Hall, D. C. Maus, G. J. Gerfen, S. J. Inati, L. R. Becerra, F. W. Dahlquist, R. G. Griffin, Science 1997, 276, 930-932.

[4] J. H. Ardenkjær-Larsen, B. Fridlund, A. Gram, G. Hansson, L. Hansson, M. H. Lerche, R. Servin, M. Thaning, K. Golman, Proc Natl. Acad. Sci. U. S. A. 2003, 100, 10158-10163.

[5] C. Song, K.-N. Hu, C.-G. Joo, T. M. Swager, R. G. Griffin, J. Am. Chem. Soc. 2006, 128, 11385-11390.

[6] S. E. Day, M. I. Kettunen, F. A. Gallagher, D.-E. Hu, M. Lerche, J. Wolber, K. Golman, J. H. Ardenkjær-Larsen, K. M. Brindle, Nat. Med. 2007, 13, 1382-1387.

[7] V. S. Bajaj, M. L. Mak-Jurkauskas, M. Belenky, J. Herzfeld, R. G Griffin, Proc. Natl. Acad. Sci. U. S. A. 2009, 106, 9244-9249.

[8] A. J. Rossini, A. Zagdoun, M. Lelli, A. Lesage, C. Copéret, L. Emsley, Acc. Chem. Res. 2013, 46, 1942-1951.

[9] J. M. Franck, A. Pavlova, J. A. Scott, S. Han, Prog. Nucl. Magn. Reson. Spectrosc. 2013, 74, 33-56.

[10] S. Jannin, J.-N. Dumez, P. Giraudeau, D. Kurzbach, J. Magn. Reson. 2019, 305, 41-50.

[11] A. Ajoy, K. Liu, R. Nazaryan, X. Lv, P. R. Zangara, B. Safvati, G. Wang, D. Arnold, G. Li, A. Lin, P. Raghavan, E. Druga, S. Dhomkar, D. Pagliero, J. A. Reimer, D. Suter, C. A. Meriles, A. Pines, Sci. Adv. 2018, 4, eaar5492.

[12] A. Henstra, P. Dirksen, W. Th. Wenckebach, Phys. Lett. A 1988, 134, 134-136.

[13] A. Henstra, T.-S. Lin, J. Schmidt, W. Th. Wencekbach, Chem. Phys. Lett. 1990, 165, 6-10.

M. linuma, Y. Takahashi, I. Shake, M. Oda, A. Masaike, T. Yabuzaki, H. M. Shimizu, J. Magn. Reson. 2005, 175, 235-241. K. Takeda, Triplet State Dynamic Nuclear Polarization, VDM Verlag Dr. Müeller:, Saarbrücken, Germany, 2009.

K. Tateishi, M. Negoro, A. Kagawa, M. Kitagawa, Angew. Chem. Int. Ed. 2013, 52, 13307-13310.

K. Tateishi, M. Negoro, S. Nishida, A. Kagawa, Y. Morita, M. Kitagawa, Proc. Natl. Acad. Sci. U. S. A. 2014, 111, 7527-7530. K. Nishimura, H. Kouno, Y. Kawashima, K. Orihashi, S. Fujiwara, K. Tateishi, T. Uesaka, N. Kimizuka, N. Yanai, Chem. Commun. 2020, 56, 7217-7232.

[19] T. Hamachi, K. Nishimura, H. Kouno, Y. Kawashima, K. Tateishi, T. Uesaka, N. Kimizuka, N. Yanai, J. Phys. Chem. Lett. 2021, 12 2645-2650.
A. Yamauchi, S. Fujiwara, K. Nishimura, Y. Sasaki, K. Tateishi, T. Uesaka, N. Kimizuka, N. Yanai, J. Phys. Chem. A 2021, 125, 4334-4340.

[21] Y. Quan, B. van den Brandt, J. Kohlbrecher, W. Th. Wenckebach, P. Hautle, Nucl. Instrum. Methods Phys. Res. A 2019, 921, 22-26.

[22] H. Kouno, K. Orihashi, K. Nishimura, Y. Kawashima, K. Tateishi, T. Uesaka, N. Kimizuka, N. Yanai, Chem. Commun. 2020, 56, 37173720.

[23] M. Negoro, A. Kagawa, K. Tateishi, Y. Tanaka, T. Yuasa, K. Takahashi, M. Kitagawa, J. Phys. Chem. A 2018, 122, 4294-4297. A. Kagawa, M. Negoro, R. Ohba, N. Ichijo, K. Takamine, Y. Nakamura, T. Murata, Y. Morita, M. Kitagawa, J. Phys. Chem. A 2018, 122, 9670-9675.

[25] W. Cao, W. D. Wang, H.-S. Xu, I. V. Sergeyev, J. Struppe, X. Wang, F. Mentink-Vigier, Z. Gan, M.-X. Xiao, L.-Y. Wang, G.-P Chen, S.-Y. Ding, S. Bai, W. Wang, J. Am. Chem. Soc. 2018, 140 6969-6977.

[26] M. Cavaillès, A. Bornet, X. Jaurand, B. Vuichoud, D. Baudouin, M. Baudin, L. Veyre, G. Bodenhausen, J.-N. Dumez, S. Jannin, C. Copéret, C. Thieuleux, Angew. Chem. Int. Ed. 2018, 57, 74537457.

[27] M. Juramy, R. Chèvre, P. C. Vioglio, F. Ziarelli, E. Besson, S. Gastaldi, S. Viel, P. Thureau, K. D. M. Harris, G. Mollica, J. Am. Chem. Soc. 2021, 143, 6095-6103.

[28] T. E. Daraï, S. F. Cousin, Q. Stern, M. Ceillier, J. Kempf, D. Eshchenko, R. Melzi, M. Schnell, L. Gremillard, A. Bornet, J Milani, B. Vuichoud, O. Cala, D. Montarnal, S. Jannin, Nat. Commun. 2021, 12, 4695

[29] S. Fujiwara, M. Hosoyamada, K. Tateishi, T. Uesaka, K. Ideta, N. Kimizuka, N. Yanai, J. Am. Chem. Soc. 2018, 140, 15606-15610.

[30] A. Pines, T. W. Shattuck, J. Chem. Phys. 1974, 61, 1255-1256.

[31] V. J. Witherspoon, J. Xu, J. A. Reimer, Chem. Rev. 2018, 118, 10033-10048.

[32] G. K. H. Shimizu, R. Vaidhyanathan, J. M. Taylor, Chem. Soc. Rev. 2009, 38, 1430-1449.

[33] S. T. Meek, J. A. Greathouse, M. D. Allendorf, Adv. Mater. 2011 23, 249-267.

[34] J.-R. Li, J. Sculley, H.-C. Zhou, Chem. Rev. 2012, 112, 869-932

[35] H. Furukawa, K. E. Cordova, M. O'Keeffe, O. M. Yaghi, Science 2013, 341, 1230444.

[36] P. Ramaswamy, N. E. Wong, G. K. H. Shimizu, Chem. Soc. Rev. 2014, 43, 5913-5932.

[37]

H.-C. Zhou, S. Kitagawa, Chem. Soc. Rev. 2014, 43, 5415-5418. G. Férey, C. Serre, Chem. Soc. Rev. 2009, 38, 1380-1399. S. Horike, S. Shimomura, S. Kitagawa, Nat. Chem. 2009, 1, 695704. T. Bataille, G. Férey, Chem. Euro. J 2004, 10, 1373-1382. Y. Liu, J.-H. Her, A. Dailly, A. J. Ramirez-Cuesta, D. A. Neumann, C. M. Brown, J. Am. Chem. Soc. 2008, 130, 11813-11818. F. Millange, C. Serre, N. Guillou, G. Férey, R. I. Walton, Angew. Chem. Int. Ed. 2008, 47, 4100-4105.

H. Kouno, Y. Kawashima, K. Tateishi, T. Uesaka, N. Kimizuka, N. Yanai, J. Phys. Chem. Lett. 2019, 10, 2208-2213.

J. Viger-Gravel, C. E. Avalos, D. J. Kubicki, D. Gajan, M. Lelli, O. Ouari, A. Lesage, L. Emsley, Angew. Chem. Int. Ed. 2019, 58 7249-7253.

S. Canossa, A. Gonzalez-Nelson, L. Shupletsov, M. del Carmen Martin, M. A. Van der Veen, Chem. Euro. J 2020, 26, 3564-3570. S. Bourrelly, P. L. Llewellyn, C. Serre, F. Millange, T. Loiseau, G. Férey, J. Am. Chem. Soc. 2005, 127, 13519-13521. N. Chanut, A. Ghoufi, M.-V. Coulet, S. Bourrelly, B. Kuchta, G. Maurin, P. L. Llewellyn, Nat. Commun. 2020, 11, 1216. G. de Combarieu, M. Morcrette, F. Millange, N. Guillou, J. Cabana, C. P. Grey, I. Margiolaki, G. Férey, J.-M. Tarascon, Chem. Mater.

2009, 21, 1602-1611.

S. Krause N. Hosono, J. Magn. Reson. 2006, 178, 42-55. 59, 15325-15341. no, S. Kitagawa, Angew. Chem. Int. Ed. 2020, K. R. Keshari, D. M. Wilson, Chem. Soc. Rev. 2014, 43, 16271659. 